\title{
GAUSSIAN PROCESSES AND HAMMERSTEIN INTEGRAL EQUATIONS
}

\author{
BY \\ MARTIN PINCUS
}

1. Introduction. In his thesis M. Schilder [1] has proven an analogue of the classical Laplace asymptotic formula for Weiner integrals. It is the purpose of this paper to generalize this formula to expectations on a large class of Gaussian processes, and to demonstrate a close connection with Hammerstein integral equations.

We say that $\rho(\sigma, \tau), 0 \leqq \sigma \leqq \tau \leqq t$, is a covariance function if $\rho(\sigma, \tau)=\rho(\tau, \sigma)$ and if for any finite set $0<\tau_{1}<\cdots<\tau_{n}<t$ the matrix $\left[\rho\left(\tau_{i}, \tau_{j}\right)\right]$ is nonnegative definite.

A Gaussian process is determined by a covariance function $\rho(\sigma, \tau), 0 \leqq \sigma \leqq \tau \leqq t$, and a mean function $\mu(\tau), 0 \leqq \tau \leqq t$. Unless explicitly stated otherwise, we shall assume that the mean function is identically zero.

If the covariance function $\rho(\sigma, \tau)$ is such that

$$
\int_{0}^{t} \int_{0}^{t} \rho(\sigma, \tau)^{2} d \sigma d \tau<\infty
$$

and is positive definite, then it defines a positive definite Hilbert-Schmidt operator $A$, through the equation

$$
(A x)(\sigma)=\int_{0}^{t} \rho(\sigma, \tau) x(\tau) d \tau, \quad x \in L^{2},
$$

where $L^{2}$ is Hilbert space of functions $x$ on $[0, t]$ with norm

$$
(x, x)^{1 / 2}=\left[\int_{0}^{t} x^{2}(\tau) d \tau\right]^{1 / 2}
$$

We shall denote by $\left\{u_{i}(\cdot)\right\}$ the normalized eigenfunctions and by $\left\{\rho_{i}\right\}$ the reciprocal eigenvalues, ordered in increasing magnitude, of the operator $A$.

When $\rho(\sigma, \tau)$ is continuous and positive definite, and if $\left\{\alpha_{i}\right\}$ is a sequence of independent Gaussian random variables with mean 0 and variance 1 , then $[5, \mathrm{pp}$. 30-34, also §5] there exists a Gaussian process with sample paths $x(\tau), 0 \leqq \tau \leqq t$, represented by

$$
x(\tau)=\sum_{i=1}^{\infty} \frac{\alpha_{i}}{\sqrt{ } \rho_{i}} u_{i}(\tau)
$$

except possibly on a $\tau$ set of Lebesgue measure 0 .

Received by the editors January 11, 1967. 
Using this representation we shall present a heuristic formalism for manipulating Gaussian expectations which makes many of the theorems about such expectations formally transparent.

Let $\left.E_{x\{}^{o\{}\right\}$ denote the expectation on the Gaussian process with covariance function $\rho(\sigma, \tau)$ and sample paths $x(\tau)$, let $E\{\}$ denote the expectation over infinite product space with the measure generated by $\alpha_{i}$ on each real line, and let $E_{n}\{\}$ denote the expectation with respect to the finite product measure generatec' by $\left\{\alpha_{i}\right\}_{i=1}^{n}$.

It follows from [5, pp. 30-31] that

$$
E_{x}^{o}\{G(x)\}=E\left\{G\left(\sum_{i=1}^{\infty} \frac{\alpha_{i}}{\sqrt{ } \rho_{i}} u_{i}(\cdot)\right)\right\}
$$

for every functional $G(\cdot)$ for which one side of the above equality exists. From the Fubini-Jessen theorem [4] we then obtain

$$
\begin{aligned}
& E_{x}^{o}\{G(x)\}=E\left\{G\left(\sum_{i=1}^{\infty} \frac{\alpha_{i}}{\sqrt{ } \rho_{i}} u_{i}(\cdot)\right)\right\} \\
& =\operatorname{lam}_{n \rightarrow \infty} E_{n}\left\{G\left(\sum_{i=1}^{n} \frac{\alpha_{i}}{\sqrt{ } \rho_{i}} u_{i}(\cdot)+\sum_{n+1}^{\infty} \frac{\alpha_{i}}{\sqrt{ } \rho_{i}} u_{i}(\cdot)\right)\right\} \\
& ={ }_{\text {a.e. }} \lim _{n \rightarrow \infty} \frac{1}{(2 \pi)^{n / 2}} \int_{-\infty}^{\infty} \cdots \int_{-\infty}^{\infty} G\left(\sum_{i=1}^{n} \frac{\alpha_{i}}{\sqrt{ } \rho_{i}} u_{i}(\cdot)+\sum_{i=n+1}^{\infty} \frac{\alpha_{i}}{\sqrt{ } \rho_{i}} u_{i}(\cdot)\right) \\
& \times \exp \left(-\frac{1}{2} \sum_{i=1}^{n} \alpha_{i}^{2}\right) \prod_{i=1}^{n} d \alpha_{i} \\
& =\text { a.e. } \lim _{n \rightarrow \infty}\left[\prod_{i=1}^{n} \rho_{i} /(2 \pi)^{n}\right]^{1 / 2} \int_{-\infty}^{\infty} \cdots \int_{-\infty}^{\infty} G\left(\sum_{i=1}^{n} \eta_{i} u_{i}(\cdot)+\sum_{i=n+1}^{\infty} \frac{\alpha_{i}}{\sqrt{ } \rho_{i}} u_{i}(\cdot)\right) \\
& \times \exp \left(-\frac{1}{2} \sum_{i=1}^{n} \rho_{i} \eta_{i}^{2}\right) \prod_{i=1}^{n} d \eta_{i}
\end{aligned}
$$

where a.e. means almost everywhere with respect to the product measure generated by $\left\{\alpha_{i}\right\}$.

Letting $x_{n}(\cdot)=\sum_{i=1}^{n} \eta_{i} u_{i}(\cdot)$ and noticing that $\sum_{i=1}^{n} \eta_{i}^{2} \rho_{i}=\left(A^{-1 / 2} x_{n}, A^{-1 / 2} x_{n}\right)$ we obtain from (1.1) that .

$$
\begin{aligned}
E_{x}^{\rho}\{G(x)\}= & \lim _{\text {a.e. }}\left(\frac{\prod_{i=1}^{n} \rho_{i}}{(2 \pi)^{n}}\right)^{1 / 2} \int_{-\infty}^{\infty} \cdots \int_{-\infty}^{\infty} G\left(x_{n}(\cdot)+\sum_{i=n+1}^{\infty} \frac{\alpha_{i}}{\sqrt{\rho_{i}}} u_{i}(\cdot)\right) \\
& \times \exp \left(-\frac{1}{2}\left(A^{-1 / 2} x_{n}, A^{-1 / 2} x_{n}\right)\right) \prod_{i=1}^{n} d \eta_{i} .
\end{aligned}
$$


Proceeding heuristically, we write

$$
\begin{array}{r}
\lim _{n \rightarrow \infty} G\left(x_{n}(\cdot)+\sum_{i=n+1}^{\infty} \frac{\alpha_{i}}{\sqrt{\rho_{i}}} u_{i}(\cdot)\right) \exp \left(-\frac{1}{2}\left(A^{-1 / 2} x_{n}, A^{-1 / 2} x_{n}\right)\right) \\
"=" G(x) \exp \left(-\frac{1}{2}\left(A^{-1 / 2} x, A^{-1 / 2} x\right)\right), \\
\lim _{n \rightarrow \infty}\left(\frac{\prod_{i=1}^{n} \rho_{i}}{(2 \pi)^{n}}\right)^{1 / 2} \int_{-\infty}^{\infty} \cdots \int_{-\infty}^{\infty} \prod_{i=1}^{n} d \eta_{i} "="[\delta x
\end{array}
$$

where the symbol $\int \delta x$ represents a translation invariant integral in function space, and is called the flat integral.

Actually, neither limit (1.3) or (1.4) exists separately. However, as a useful formal device we write

$$
E_{x}^{o}\{G(x)\} \text { " }="\left[G(x) \exp \left(-\frac{1}{2}\left(A^{-1 / 2} x, A^{-1 / 2} x\right)\right) \delta x\right.
$$

where the right hand side is to be treated as a one dimensional Riemann integral.

The classical Laplace asymptotic formula may be written in the form

$$
\lim _{\lambda \rightarrow 0} \frac{\int_{-\infty}^{\infty} G(x) \exp \left(-F(x) / \lambda^{2}\right) d x}{\int_{-\infty}^{\infty} \exp \left(-F(x) / \lambda^{2}\right) d x}=G\left(x^{*}\right)
$$

where $F(x),-\infty<x<\infty$, is assumed continuous and to have a unique global minimum at $x^{*}$. Furthermore, $G$ is assumed continuous at $x^{*}$.

Let $G(\cdot)$ and $F(\cdot)$ now denote functionals defined on the sample paths of a Gaussian process with covariance function $\rho(\sigma, \tau)$. Using the flat integral, a formula analogous to (1.6) is

$$
\begin{aligned}
\lim _{\lambda \rightarrow 0} \frac{\Gamma G(x) \exp \left(-\left\{\frac{1}{2}\left(A^{-1 / 2} x, A^{-1 / 2} x\right)+F(x)\right\} / \lambda^{2}\right) \delta x}{\int \exp \left(-\left\{\frac{1}{2}\left(A^{-1 / 2} x, A^{-1 / 2} x\right)+F(x)\right\} / \lambda^{2}\right) \delta x} \\
=\lim _{\lambda \rightarrow 0} \frac{\left\lceil G(\lambda x) \exp \left(-\left\{F(\lambda x)-\frac{1}{2}\left(A^{-1 / 2} x, A^{-1 / 2} x\right)\right\} / \lambda^{2}\right) \delta x\right.}{\int \exp \left(-\left\{F(\lambda x)-\frac{1}{2}\left(A^{-1 / 2} x, A^{-1 / 2} x\right)\right\} / \lambda^{2}\right) \delta x} \\
=\lim _{\lambda \rightarrow 0} \frac{E_{x\{}^{o}\left\{G(\lambda x) \exp \left(-F(\lambda x) / \lambda^{2}\right)\right\}}{E_{x}^{o}\left\{\exp \left(-F(\lambda x) / \lambda^{2}\right)\right\}}=G\left(x^{*}\right),
\end{aligned}
$$

where it is assumed that the functional $\frac{1}{2}\left(A^{-1 / 2} x, A^{-1 / 2} x\right)+F(x)$ has a unique global minimum at $x^{*}$. The last equality in (1.7) is the conclusion of our main theorem which is stated more precisely in Theorem 4.1.

$\$ 5$ contains the applications of Theorem 4.1 to Hammerstein integral equations. Under the conditions of Theorem 5.1, we obtain a closed form solution of the Hammerstein integral equation

$$
x(\sigma)+\int_{0}^{t} \rho(\sigma, \tau) f(\tau, x(\tau)) d \tau=0 .
$$


The representation obtained is the limit of ratio of two expectations and makes Hammerstein's original conditions for proving existence of a solution to the above integral equation appear naturally as integrability conditions. The last part of $\$ 5$ deals with the case of Brownian motion, i.e., $\rho(\sigma, \tau)=\min (\sigma, \tau)$. Through the Feynman-Kac formula [9]-[11] we are able to relate the solution of the above integral equation, with a kernel closely related to $\min (\sigma, \tau)$, to the Green's function of a linear parabolic equation. In this case the Hammerstein integral equation is equivalent to an ordinary differential equation.

$\$ 2$ contains auxiliary lemmas needed in the proof of Theorem 4.1 .

$\$ 3$ contains a Gaussian type estimate on the distribution of the supremum under certain conditions on the covariance function. It is this estimate that is used as a hypothesis in the statement of Theorem 4.1 in $\$ 4$.

ACKNOWLedgments. The author would like to thank his thesis advisor, Professor M. Donsker, for his valuable help, and Professor S. Varadhan for suggesting the proof of Lemma 3.1 as well as many helpful discussions.

2. Properties of $H(x)=\frac{1}{2}\left(A^{-1 / 2} x, A^{-1 / 2} x\right)+F(x)$. In this section we define the derivative of a functional and demonstrate some properties of $H(x)$ needed for the proof of the theorem in $\$ 4$. The last part of this section shows the integrability of certain functionals.

Let $C$ be the space of continuous functions on $[0, t]$ with norm, $\|x\|$ $=\sup _{0 \leqq \tau \leqq t}|x(\tau)|$, and let $L^{2}$ be the Hilbert space of functions on $[0, t]$ with norm

$$
(x, x)^{1 / 2}=\left[\int_{0}^{t} x^{2}(\tau) d \tau\right]^{1 / 2}
$$

Let $F(x)$ be a real valued functional defined on $C$. If $\Phi \in C$, then the derivative of $F$ in the direction $\Phi$ at $x$ is

$$
\lim _{\varepsilon \rightarrow 0} \frac{F(x+\varepsilon \Phi)-F(x)}{\varepsilon}
$$

whenever this limit exists.

If there exists a bounded linear functional $T$ such that the derivative of $F$ in the direction $\Phi$ at $x$ is given by $T(\Phi)$, then $T$ is called the Gateaux differential or variation of $F$ at $x$.

When $T$ is also continuous in the $L^{2}$ topology it follows from the Reisz representation theorem that there exists an element of $L^{2}, \delta F(x) / \delta x(\tau)$, such that

$$
T(\Phi)=\int_{0}^{t} \frac{\delta F(x)}{\delta x(\tau)} \Phi(\tau) d \tau
$$

In this case $\delta F(x) / \delta x(\tau)$ is called the derivative of $F$ with respect to $x$ at the point $\tau$. 
In what follows $\rho(\sigma, \tau), 0 \leqq \sigma, \tau \leqq t$, will denote a continuous, symmetric, positive definite kernel. $A$ will denote the Hilbert-Schmidt operator defined by $\rho(\sigma, \tau)$, i.e.

$$
(A x)(\sigma)=\int_{0}^{t} \rho(\sigma, \tau) x(\tau) d \tau, \quad x \in L^{2} .
$$

We remark that $A$ is a compact, self adjoint, positive definite operator on $L^{2}$ with reciprocal eigenvalues $\left\{\rho_{i}\right\}, \rho_{i}>0$, and normalized eigenfunctions $\left\{u_{i}(\sigma)\right\}$.

LEMMA 1. $A^{1 / 2}$ is a Hilbert-Schmidt operator with kernel $K(\sigma, \tau)$ and is a completely continuous mapping of $L^{2}$ into $C$ [4].

LEMMA 2. (a) $\left\|A^{1 / 2} x\right\|^{2} \leqq M(x, x), M=\sup _{0 \leqq \sigma \leqq t} \rho(\sigma, \sigma)$.

(b) $(A x, x) \leqq(x, x) / \rho_{1}$ where $1 / \rho_{1}$ is the largest eigenvalue of $A$.

(c) $(A x, A x) \leqq(A x, x) / \rho_{1}$.

(d) $\|A x\|^{2} \leqq M(x, x) / \rho_{1}[4]$.

Let $D\left(A^{-1}\right)$ denote the domain of the operator $A^{-1}$. We now introduce a new Hilbert space, $L_{A}^{2}$, defined as the Cauchy completion of the space $D\left(A^{-1}\right)$ under the norm, $\left(A^{-1} x, x\right)^{1 / 2}$.

LEMMA 3. $L_{A}^{2}=D\left(A^{1 / 2}\right)[3]$.

Let $[x, y]_{A}$ denote the inner product on $L_{A}^{2}$. From Lemma 3 it is clear that $[x, x]_{A}=\left(A^{-1 / 2} x, A^{-1 / 2} x\right)$. We also note that in terms of $L_{A}^{2}$ Lemma 1 can be rephrased to read that every bounded set in $L_{A}^{2}$ is precompact in $C$.

LEMMA 4. Let $F(x)$ be a continuous functional on $C$ satisfying

$$
F(x) \geqq-\frac{1}{2} c_{1}(x, x)-c_{2}, \quad c_{1}<\rho_{1}, c_{2} \text { any real number. }
$$

It then follows that there exists at least one point $x^{*} \in D\left(A^{-1 / 2}\right)$ at which

$$
H(x)=\frac{1}{2}\left(A^{-1 / 2} x, A^{-1 / 2} x\right)+F(x)
$$

assumes its global minimum value for all $x \in C$.

Let $B$ be the set of points at which $H(x)$ assumes its global minimum, and let $\left\{x_{n}\right\}$ be a minimizing sequence of $H(x)$. We then have $B \subset D\left(A^{-1 / 2}\right)$ and that there exists a subsequence $\left\{x_{n_{i}}\right\}$ of $\left\{\dot{x}_{n}\right\}$ such that $\left\{x_{n_{1}}\right\}$ converges uniformly to a point $x^{*} \in B$.

Proof. By Lemma 2.2(b) it follows that

$$
\frac{1}{2}\left(A^{-1 / 2} x, A^{-1 / 2} x\right)+F(x) \geqq+\frac{1}{2}\left(\rho_{1}-c_{1}\right)(x, x)-c_{2} \geqq-c_{2} .
$$

Thus, $H(x)$ is bounded below. Assume that the global minimum of $H$ is zero. It is also clear when $\left(A^{-1 / 2} x_{n}, A^{-1 / 2} x_{n}\right) \rightarrow \infty$ that

$$
\frac{1}{2}\left(A^{-1 / 2} x_{n}, A^{-1 / 2} x_{n}\right)+F\left(x_{n}\right) \rightarrow \infty \quad \text { as } n \rightarrow \infty .
$$


Therefore, the sequence $\left\{\left(A^{-1 / 2} x_{n}, A^{-1 / 2} x_{n}\right)=\left[x_{n}, x_{n}\right]_{A}\right\}$ is bounded. By the comments following Lemma 3 we see that $\left\{x_{n}\right\}$ contains a subsequence $\left\{x_{n_{1}}\right\}$ which forms a Cauchy sequence in $C$. Let $\lim _{i \rightarrow \infty} x_{n_{1}}=y \in C$. We shall show $y \in D\left(A^{-1 / 2}\right)$.

We note that $\left\{x_{n_{i}}\right\}$ forms a bounded set in $L_{A}^{2}$. Since every bounded set in Hilbert space is weakly precompact [4], and since Hilbert space is weakly complete, we see that there exists a subsequence of $\left\{x_{n_{t}}\right\}$ which converges weakly in $L_{A}^{2}$ to a point $u \in L_{A}^{2}$. To save on notation we shall denote this subsequence as $\left\{x_{n_{1}}\right\}$ also.

By definition of weak convergence we see that

$$
\left[z, x_{n_{i}}\right]_{A} \rightarrow[z, u]_{A} \text { as } i \rightarrow \infty \text { for all } z \in L_{A}^{2} .
$$

In particular, when $z \in D\left(A^{-1}\right)$ we have

$$
\left[z, x_{n_{1}}-u\right]_{A}=\left(A^{-1} z, x_{n_{i}}-u\right) \rightarrow 0 \quad \text { as } i \rightarrow \infty .
$$

Since $\left\{x_{n_{i}}\right\}$ converges uniformly to $y$ it also follows that if $z \in D\left(A^{-1}\right)$ then

$$
\left|\left(A^{-1} z, x_{n_{i}}-y\right)\right|^{2} \leqq\left(A^{-1} z, A^{-1} z\right)\left(x_{n_{1}}-y, x_{n_{t}}-y\right) \rightarrow 0 \quad \text { as } i \rightarrow \infty .
$$

Therefore, we have

$$
\lim _{i \rightarrow \infty}\left[z, x_{n_{i}}\right]_{A}=\left(A^{-1} z, u\right)=\left(A^{-1} z, y\right) \text { for all } z \in D\left(A^{-1}\right)
$$

which implies that $u=y \in D\left(A^{-1 / 2}\right)$.

In any normed linear space the norm is weakly lower semicontinuous [4], i.e. $x_{n} \rightarrow x$ weakly implies

$$
|x| \leqq \lim _{n} \inf \left|x_{n}\right| \quad(|x|=\operatorname{norm} x) .
$$

Applying this to $L_{A}^{2}$, we write $H(x)=\frac{1}{2}[x, x]_{A}+F(x)$ and obtain

$$
0=\lim _{i \rightarrow \infty} H\left(x_{n_{i}}\right)=\lim _{i} \inf \left(\frac{1}{2}\left[x_{n_{t}}, x_{n_{t}}\right]_{A}+F\left(x_{n_{t}}\right)\right) \geqq \frac{1}{2}[y, y]_{A}+F(y) \geqq 0 .
$$

Therefore, $\frac{1}{2}\left(A^{-1 / 2} y, A^{-1 / 2} y\right)+F(y)=0$ which implies that $y=x^{*}$ for some $x^{*} \in B$.

Lemma 5. Let $F(x)$ satisfy the conditions of Lemma 4. Assume that $x^{*}$ is the only point of $B$ that satisfies $\left\|x-x^{*}\right\| \leqq R$, and that $H\left(x^{*}\right)=0$. It then follows that, given $\delta>0$ there exists a $\theta(\delta)>0$ such that $\delta<\left\|x-x^{*}\right\| \leqq R$ implies $H(x) \geqq \theta(\delta)$.

Proof. From Lemma 4 it follows that every minimizing sequence $\left\{x_{n}\right\}$ that converges in $C$ and satisfies $\left\|x_{n}-x^{*}\right\| \leqq R$ must converge to $x^{*}$.

Suppose there exists a $\delta>0$ such that $\delta<\left\|x-x^{*}\right\| \leqq R$ implies $H(x) \leqq \theta$ for all $\theta>0$. But this implies there exists a minimizing sequence $\left\{x_{n}\right\}$ such that $\delta \leqq\left\|x_{n}-x^{*}\right\|$ $\leqq R$. The first comment shows this is a contradiction.

LEMMA 6. If $H(x)$ has a global minimum at $x^{*}, H\left(x^{*}\right)=0$, and $F$ is a continuous functional on $C$, then

(a) $\inf _{x \in L^{2}}\left[\frac{1}{2}(A x, x)+F(A x)\right]=0$.

(b) Given $\eta>0$ there exists a $\beta>0$ such that $\left(A^{1 / 2} x-A^{-1 / 2} x^{*}, A^{1 / 2} x-A^{-1 / 2} x^{*}\right)<\beta$ implies $\frac{1}{2}(A x, x)+F(A x)<\eta$. 
Proof. If we write $y=A x$, then for $x \in L^{2}$,

$$
\frac{1}{2}(A x, x)+F(A x)=\frac{1}{2}\left(A^{-1} y, y\right)+F(y), \quad y \in D\left(A^{-1}\right) .
$$

Since $D\left(A^{-1}\right)$ is dense in $L_{A}^{2}=D\left(A^{-1 / 2}\right)$, and convergence in $L_{A}^{2}$ implies uniform convergence, we have part (a) of the lemma.

From the fact that $H\left(x^{*}\right)=0$, it follows that

$$
\frac{1}{2}(A x, x)+F(A x) \leqq \frac{1}{2}\left|(A x, x)-\left(A^{-1 / 2} x^{*}, A^{-1 / 2} x^{*}\right)\right|+\left|F(A x)-F\left(x^{*}\right)\right| .
$$

By Lemma 2(a) we have

$$
\left\|A x-x^{*}\right\|=\left\|A^{1 / 2}\left(A^{1 / 2} x-A^{-1 / 2} x^{*}\right)\right\| \leqq M \beta .
$$

Therefore part (b) follows from the continuity of the inner product on $L^{2}$ and the continuity of $F$ on $C$.

LEMMA 7. If $\rho(\sigma, \tau)$ is continuous and if $b>0$,

$$
E_{x}^{o}\{G(x)\}=D_{\rho}(-b) E_{x}^{o}\left\{G(x+b A x) \exp \left[-\frac{1}{2} b^{2}(A x, x)-b(x, x)\right]\right\}
$$

for all integrable functionals $G(x)$, where $D_{\rho}$ is the Fredholm determinant of $\rho(\sigma, \tau)$.

We refer to [5] for the proof. Here we note that if one side of the above equality exists so does the other and they are equal.

LEMMA 8. Suppose $\rho(\sigma, \tau), 0 \leqq \sigma, \tau \leqq t$, is continuous and there is a corresponding Gaussian process with continuous sample paths, $x(\tau), 0 \leqq \tau \leqq t$. Further, suppose that if $a>h>0$ then

$$
P\{\|x\| \geqq a\} \leqq c \exp \left(-\gamma a^{2}\right),
$$

where $c, \gamma>0$ depend only on $h$. Suppose $F$ and $G$ are real valued, measurable functionals defined on $C$ satisfying

$$
\begin{gathered}
|G(x)|<\exp \left(c\|x\|^{2}\right), \quad c>0, \\
F(x) \geqq-\frac{1}{2} c_{1}(x, x)-c_{2}, \quad c_{1}<\rho_{1}, c_{2} \text { any real number. }
\end{gathered}
$$

If

$$
0<\lambda<\min \left(\frac{\frac{1}{2}\left(1-c_{1} / \rho_{1}\right)}{\left(c_{1} / 2+M c / \rho_{1}\right)},\left(\frac{\gamma}{2 c}\right)^{1 / 2}\right)
$$

then

$$
\begin{aligned}
& E_{x}^{\rho}\left\{|G(\lambda x)| \exp \left(-F(\lambda x) / \lambda^{2}\right)\right\} \\
& =D_{\rho}\left(-\frac{1}{\lambda}\right) E_{x}^{o}\left\{|G(\lambda x+A x)| \exp \left(-\left\{\left(\frac{1}{2} A x+\lambda x, x\right)+F(A x+\lambda x)\right\} / \lambda^{2}\right)\right\}
\end{aligned}
$$

is finite. 
Proof. The equality in (2.4) follows from Lemma 7, using $b=1 / \lambda$. Using (2.2) and (2.3) we have

$$
\begin{aligned}
E_{x}^{o}\{|G(\lambda x+A x)| & \left.\exp \left(-\left\{\left(\frac{1}{2} A x+\lambda x, x\right)+F(A x+\lambda x)\right\} / \lambda^{2}\right)\right\} \\
\leqq & E_{x}^{o}\left\{\exp \left(c \lambda^{2}\|x\|^{2}+2 c \lambda\|x\|\|A x\|+c\|A x\|^{2}\right)\right. \\
& \left.\times \exp \left(-\left\{\left(\frac{1}{2} A x+\lambda x, x\right)-c_{1}(A x+\lambda x, A x+\lambda x)-c_{2}\right\} / \lambda^{2}\right)\right\}
\end{aligned}
$$

By Lemma 2(a), (b), (c) and (d) we have

$$
\begin{aligned}
\frac{1}{2}(A x & +\lambda x, x)-\frac{1}{2} c_{1}(A x+\lambda x, A x+\lambda x)-\lambda^{2} c\|A x\|^{2} \\
& =\frac{1}{2}(A x, x)-\frac{1}{2} c_{1}(A x, A x)+\lambda\left[(x, x)-c_{1}(A x, x)\right]-\lambda^{2}\left[\frac{1}{2} c_{1}(x, x)+c\|A x\|^{2}\right] \\
& \geqq \frac{1}{2}\left(1-c_{1} / \rho_{1}\right)(A x, x)+\lambda\left(1-c_{1} / \rho_{1}\right)(x, x)-\lambda^{2}\left[c_{1} / 2+M c / \rho_{1}\right](x, x) \\
& =\frac{1}{2}\left(1-c_{1} / \rho_{1}\right)(A x, x)+\lambda\left[\left(1-c_{1} / \rho_{1}\right)-\lambda\left(c_{1} / 2+M c / \rho_{1}\right)\right](x, x)
\end{aligned}
$$

which by our choice of $\lambda$ is

$$
\geqq \frac{1}{2}\left(1-c_{1} / \rho_{1}\right)(A x, x)+\lambda \frac{1}{2}\left(1-c_{1} / \rho_{1}\right)(x, x) \geqq 0 .
$$

Using (2.6), Lemma 2(d) and our choice of $\lambda$, we see that the right hand side of (2.5) is

$$
\leqq \exp \left(-c_{2} / \lambda^{2}\right) E_{x}^{o}\left\{\exp \left(\gamma / 2\|x\|^{2}+2\left(c \gamma M / 2 \rho_{1}\right)^{1 / 2}\|x\|^{3 / 2}\right)\right\} .
$$

If we write $f(u)=P\{\|x\|<u\}$ then the integral in (2.7) can be written as

$$
\int_{0}^{\infty} \exp \left(\frac{\gamma}{2} u^{2}+2\left(\frac{c \gamma M}{2 \rho_{1}}\right)^{1 / 2} u^{3 / 2}\right) d F(u)
$$

which by (2.1) is finite.

LEMMA 9. If the covariance function $\rho(\sigma, \tau), 0 \leqq \sigma, \tau \leqq t$, is continuous, $0<\alpha \leqq 1$, $K>0$ and $0<\lambda \leqq 1$, then

$$
E_{x}^{o}\left\{\exp \left(+\frac{K(x, x)^{\alpha / 2}}{\lambda^{2-\alpha}}-\frac{(x, x)}{\lambda}\right)\right\} \leqq 2 \exp \left(\frac{K^{2 /(2-\alpha)}}{\lambda^{(2-\alpha) / 2}}\right) .
$$

Proof. Let $F(u)=P\left((x, x)^{1 / 2}<u\right)$. Then

$$
\begin{aligned}
& E_{x}^{p}\left\{\exp \left(K(x, x)^{\alpha / 2} / \lambda^{2-\alpha}-(x, x) / \lambda\right)\right\}=\int_{0}^{\infty} \exp \left(K u^{\alpha} / \lambda^{2-\alpha}-u^{2} / \lambda\right) d F(u) \\
& \leqq \int_{0}^{Z^{1 /(2-\alpha) / \lambda(1-\alpha) /(2-\alpha)}} \exp \left(K u^{\alpha} / \lambda^{2-\alpha}-u^{2} / \lambda\right) d F(u)+1 \\
& \leqq \exp \left(K^{2 /(2-\alpha)} / \lambda^{(4-3 \alpha) /(2-\alpha)}\right)+1 \\
& \leqq 2 \exp \left(K^{2 /(2-\alpha)} / \lambda^{((2-\alpha) / 2)}\right)
\end{aligned}
$$

3. An estimate on the distribution of the supremum. The proof of the principal theorem (\$4) applies only to those Gaussian processes with continuous sample paths $x(\tau)$, and such that if $a>h>0$, then

$$
P(\|x\| \geqq a) \leqq c \exp \left(-\gamma a^{2}\right)
$$

where $c, \gamma>0$ depend only on $h$. 
In this section we state two conditions on the kernel $\rho(\sigma, \tau)$ which are sufficient to ensure that the above requirements are fulfilled.

By a heuristic argument using the flat integral we should expect this result to be true whenever $\rho(\sigma, \tau), 0 \leqq \sigma, \tau \leqq t$, and the sample paths $x(\tau)$ are continuous by using the flat integral [12].

In the first lemma below, we assume $\rho(\sigma, \tau), 0 \leqq \sigma, \tau \leqq t$, satisfies a Hölder condition of order $\alpha$. It then follows as a special case of a theorem of Kolmogorov [6], that there is a Gaussian process generated by $\rho(\sigma, \tau)$ with continuous sample paths $x(\tau), 0 \leqq \tau \leqq t$. In fact, as Ciesielski [7] has shown, the sample paths are Hölder continuous of order $\beta, \beta<\alpha / 2$.

The proof of Lemma 1 closely follows Prohorov's proof [8] of Kolmogorov's theorem, the principal difference being we use Gaussian type estimates where Prohorov, in the more general setting of that paper, is only able to use Tchebycheff type inequalities. See [12] for proofs.

LEMMA 1. If the symmetric, positive definite kernel $\rho(\sigma, \tau), 0 \leqq \sigma, \tau \leqq t$, satisfies

$$
\left|\rho(\sigma, \tau)-\rho\left(\sigma^{\prime}, \tau\right)\right| \leqq K\left|\sigma-\sigma^{\prime}\right|^{\alpha}, \quad K>0,0<\alpha \leqq 1,
$$

then there is a Gaussian process generated by $\rho(\sigma, \tau)$ with continuous sample paths $x(\tau), 0 \leqq \tau \leqq t$, such that if $a>\delta>0$

$$
P\{\|x\| \geqq a\} \leqq c \exp \left(-\gamma a^{2}\right)
$$

where $c$ depends only on $\delta$, and

$$
\gamma=\frac{9 \cdot 2^{4 / \ln 2}(\alpha \ln 2)^{4}}{2 \pi \cdot t^{\alpha} \cdot 4^{4} \cdot K}
$$

LEMMA 2. If $\rho(\sigma, \tau), 0 \leqq \sigma, \tau \leqq t$ is the iterate of a continuous, positive definite kernel $\rho(\sigma, \tau), 0 \leqq \sigma, \tau \leqq t$, then there is a Gaussian process generated by $\rho(\sigma, \tau)$ with continuous sample paths $x(\tau), 0 \leqq \tau \leqq t$.

Moreover, we have for $a>0$

$$
P\{\|x\|>a\}<c \exp \left(-\gamma a^{2}\right), \text { for } \gamma<\rho_{1} / 2 M, M=\sup _{0 \leqq \sigma \leqq t} K(\sigma, \sigma)
$$

and $c$ is independent of $a$ [12].

4. Proof of the Main Theorem. Before turning to the proof of our principal theorem we shall remind the reader of some notation that has been introduced.

Let $C$ denote the space of continuous functions $x$, on $[0, t]$ with norm $\|x\|$ $=\sup _{0 \leqq \sigma \leqq t}|x(\sigma)|$ and let $L^{2}$ denote the space of square integrable functions on $[0, t]$ with norm

$$
(x, x)^{1 / 2}=\left[\int_{0}^{t} x^{2}(\tau) d \tau\right]^{1 / 2}
$$


If $\rho(\sigma, t), 0 \leqq \sigma, \tau \leqq t$, is a symmetric, continuous, positive definite kernel (covariance function), we denote by $\boldsymbol{A}$ the positive definite, Hilbert-Schmidt operator defined by $\rho(\sigma, \tau)$, i.e.

$$
(A x)(\sigma)=\int_{0}^{t} \rho(\sigma, \tau) x(\tau) d \tau, \quad x \in L^{2} .
$$

The normalized eigenfunctions of $A$ are denoted by $\left\{u_{i}(\tau)\right\}$, and the reciprocal eigenvalues are denoted by $\left\{\rho_{i}\right\}$, where the sequence $\left\{p_{i}\right\}$ is ordered according to increasing magnitude. The symbol $\left.E_{x\{}^{o\{}\right\}$ denotes the expectation on the Gaussian process with covariance function $\rho(\sigma, \tau)$, mean function 0 , and whose sample paths are $x$.

THEOREM 1. Let $\rho(\sigma, \tau), 0 \leqq \sigma, \tau \leqq t$, be a continuous, symmetric, positive definite kernel for which there is a Gaussian process generated by $\rho(\sigma, \tau)$ having continuous sample paths $x(\tau), 0 \leqq \tau \leqq t\left({ }^{1}\right)$.

Assume that if $a>h>0$, then

$$
P\{\|x\| \geqq a\} \leqq c \exp \left(-\gamma a^{2}\right), \text { where } \gamma>0, c>0,
$$

depend only on $h\left({ }^{2}\right)$.

Let $F$ be a continuous, real valued functional on $C$ satisfying

$$
F(x) \geqq-\frac{1}{2} c_{1}(x, x)-c_{2}, \quad c_{1}<\rho_{1},
$$

where $\rho_{1}$ is the smallest eigenvalue of $A^{-1}$, and $c_{2}$ is real.

Let $x^{*}$ denote a point at which

$$
H(x)=\frac{1}{2}\left(A^{-1 / 2} x, A^{-1 / 2} x\right)+F(x)
$$

attains its global minimum over $x \in C\left(^{3}\right)$ and let

$$
R=\max \left\{1,\left[\left(c_{2}+1\right) \frac{2}{\gamma}\right]^{1 / 2},\left[\frac{2 M\left(\rho_{1}+\rho_{1} c_{2}\right)}{\rho_{1}-c_{1}}\right]^{1 / 2}+\gamma\left[\frac{4 M \rho_{1} c_{2}}{\rho_{1}-c_{1}}\right]^{1 / 2}\right\}
$$

Assume that

$|F(x)-F(y)| \leqq K_{1}(x-y, x-y)^{\alpha / 2}, \quad\left\|x-x^{*}\right\| \leqq 2 R,\left\|y-x^{*}\right\| \leqq 2 R, 0<\alpha \leqq 1$.

Furthermore, suppose that $x^{*}$ is the only point in the sphere $\left\{x \in C:\left\|x-x^{*}\right\| \leqq R\right\}$ at which $H(x)$ attains its global minimum.

Finally, let $G(x)$ be a measurable functional on $C$ such that $G$ is continuous at $x^{*}$ and moreover suppose

$$
|G(x)| \leqq \exp \left(c_{3}\|x\|^{2}\right), \quad c_{3}>0 .
$$

( $\left.{ }^{1}\right)$ Simple sufficient conditions for these hypotheses are given in $\$ 3$.

(2) Simple sufficient conditions for this hypothesis were given in $\$ 3$.

$\left.{ }^{3}\right)$ It is proven in $\$ 2$ that under these conditions $H(x)$ attains at least one global minimum $x^{*} \in D\left(A^{-1 / 2}\right) \subset C$. 
Under these conditions we have

$$
\lim _{\lambda \rightarrow 0} \frac{E_{x}^{o}\left\{G(\lambda x) \exp \left(-F(\lambda x) / \lambda^{2}\right)\right\}}{E_{x}^{o}\left\{\exp \left(-F(\lambda x) / \lambda^{2}\right)\right\}}=G\left(x^{*}\right) .
$$

Proof. Without loss of generality we may assume $H\left(x^{*}\right)=0$. It is also clear from Lemmas 2.7 and 2.8 that in order to prove (4.1) it is sufficient to show

$$
\lim _{\lambda \rightarrow 0} \frac{E_{x}^{o}\left\{\left|G(\lambda x+A x)-G\left(x^{*}\right)\right| \exp \left(-\left[\left(\frac{1}{2} A x+\lambda x, x\right)+F(A x+\lambda x)\right] / \lambda^{2}\right)\right\}}{E_{x}^{o}\left\{\exp \left(-\left[\left(\frac{1}{2} A x+\lambda x\right)+F(A x+\lambda x)\right] / \lambda^{2}\right)\right\}}=0 .
$$

We shall prove (4.2) by breaking up the region of integration in the numerator of the limitand in (4.2) into five subregions. Writing the limitand as a sum of five fractions we then show that for $\varepsilon>0$ each fraction can be made smaller than $\varepsilon / 5$ by choosing $\lambda$ sufficiently small.

The sets into which we break up the region of integration are:

$$
\begin{aligned}
& J_{1}^{\lambda}=\left\{x:\|\lambda x\| \leqq \frac{1}{2} \delta,\left\|A x-x^{*}\right\| \leqq \frac{1}{2} \delta\right\}, \\
& J_{2}^{\lambda}=\left\{x: \frac{1}{2} \delta<\|\lambda x\| \leqq R,\left\|A x-x^{*}\right\| \leqq R\right\}, \\
& J_{3}^{\lambda}=\left\{x:\|\lambda x\| \leqq \frac{1}{2} \delta, \frac{1}{2} \delta<\left\|A x-x^{*}\right\| \leqq R\right\}, \\
& J_{4}^{\lambda}=\left\{x: R<\|\lambda x\|,\left\|A x-x^{*}\right\| \leqq R\right\}, \\
& J_{5}=\left\{x: R<\left\|A x-x^{*}\right\|\right\},
\end{aligned}
$$

where $\delta>0$ is chosen so that

(a) $\delta<\min (1, R)$,

(b) $\theta(\delta)<1$ (where $\theta(\delta)$ is defined in Lemma 2.5),

(c) $\left\|x-x^{*}\right\|<\delta$ implies $\left|G(x)-G\left(x^{*}\right)\right|<\varepsilon / 5$,

and $\lambda>0$ is chosen so that

$$
\lambda<\min \left(1,\left[\min \left(\frac{\gamma \delta^{2}}{64}, \frac{\theta\left(\frac{1}{2} \delta\right)}{8}\right)\right]^{1 / \alpha} /(K+1)^{1 / \alpha}, \frac{1}{2} \frac{\left(1-c_{1} / \rho_{1}\right)}{c_{1} / 2+M / \rho_{1}},\left(\frac{\gamma}{4 c_{3}}\right)^{1 / 2}\right) .
$$

We introduce the following notation: $E_{x \in J}^{o}\{G(x)\}$ will denote the expectation of $G$ over the set $J$,

$$
\begin{array}{rl}
E & =E_{x}^{o}\left\{\exp \left(-\left[\left(\frac{1}{2} A x+\lambda x, x\right)+F(A x+\lambda x)\right] / \lambda^{2}\right)\right\} \\
E_{i} & =E_{x \in J_{i}^{\lambda}}^{o}\left\{\left|G(A x+\lambda x)-G\left(x^{*}\right)\right| \exp \left(-\left[\left(\frac{1}{2} A x+\lambda x, x\right)+F(A x+\lambda x)\right] / \lambda^{2}\right)\right\} \\
i=1, \ldots, 5 & i=1,
\end{array}
$$

Let

$$
\eta<\min \left(\gamma \delta^{2} / 64, \theta\left(\frac{1}{2} \delta\right) / 8\right) .
$$

By Lemma 2.6(b) we can choose $\beta>0$ so that,

$$
0<\beta<(R-(1 / 2)) / M^{1 / 2},
$$


and such that $\left(A^{1 / 2} x-A^{-1 / 2} x^{*}, A^{1 / 2} x-A^{-1 / 2} x^{*}\right)^{1 / 2}<\beta$ implies that $\frac{1}{2}(A x, x)$ $+F(A x)<\eta$. Let

$$
B_{\beta}=\left\{x:\left(A^{1 / 2} x-A^{-1 / 2} x^{*}, A^{1 / 2} x-A^{-1 / 2} x^{*}\right)^{1 / 2}<\beta,\|x\|<\frac{1}{2}\right\} .
$$

If $x \in B_{\beta}$ we have by Lemma 2.2(a),

$$
\begin{aligned}
\left\|A x+\lambda x-x^{*}\right\| & \leqq\left\|A x-x^{*}\right\|+\frac{1}{2} \leqq M^{1 / 2}\left(A^{1 / 2} x-A^{-1 / 2} x^{*}, A^{1 / 2} x-A^{-1 / 2} x^{*}\right)^{1 / 2}+\frac{1}{2} \\
& \leqq M^{1 / 2} \beta+\frac{1}{2} \quad \text { which by }(4.6) \text { is } \leqq R+\frac{1}{2} .
\end{aligned}
$$

Using the hypotheses on $F$ we then see that

$$
\begin{aligned}
E & \geqq E_{x \in B_{\beta}}^{o}\left\{\exp \left(-\frac{1}{\lambda^{2}}\left[\frac{1}{2}(A x, x)+F(A x)\right]\right) \exp \left(-\frac{1}{\lambda^{2}}[F(A x+\lambda x)-F(A x)]\right)\right. \\
& \left.\geqq \exp \left(-\frac{1}{\lambda}(x, x)\right)\right\} \\
& \geqq E_{x \in B_{\beta}}\left\{\exp \left(-\frac{\eta}{\lambda^{2}}\right) \exp \left(-\frac{K_{1}}{\lambda^{2-\alpha}}(x, x)^{\alpha / 2}\right) \exp \left(-\frac{1}{\lambda}(x, x)\right)\right\} \\
& \left.=\frac{1}{\lambda^{2}}\left\{\eta+\lambda^{\alpha} K_{1}+\lambda\right\}\right) P\left(B_{\beta}\right) .
\end{aligned}
$$

From (4.4) it follows that

$$
E \geqq \exp \left(-2 \eta / \lambda^{2}\right) P\left(B_{\beta}\right)
$$

By (4.3)(c) and the definition of $E$ we have,

$$
E_{1} \leqq \varepsilon E / 5 \text {. }
$$

Using the hypotheses on $G$ we see that

$$
\left|G(A x+\lambda x)-G\left(x^{*}\right)\right| \leqq K_{3} \exp \left(c_{3} \lambda^{2}\|x\|^{2}+2 c_{3} \lambda\|x\|\|A x\|+c_{3}\|A x\|^{2}\right)
$$

where $K_{3}$ is some positive constant. If $x \in J_{2}^{\lambda} \cup J_{3}^{\lambda}$ it follows that

$$
\left\|A x+\lambda x-x^{*}\right\| \leqq\left\|A x-x^{*}\right\|+\|\lambda x\| \leqq 2 R,
$$

and that $\|A x\|$ and $\|\lambda x\|$ are each bounded individually.

Therefore, (4.9) implies that for some positive constant $K_{4}$

$$
\left|G(A x+\lambda x)-G\left(x^{*}\right)\right| \leqq K_{4}, \quad x \in J_{2}^{\lambda} \cup J_{3}^{\lambda},
$$

while the hypotheses on $F$ show

$$
|F(A x+\lambda x)-F(A x)| \leqq K_{1}(x, x)^{\alpha / 2}, \quad x \in J_{2}^{\lambda} \cup J_{3}^{\lambda} .
$$

Using (4.10) and (4.11) we see

$$
E_{2} \leqq K_{4} E_{x \in J_{2}^{\lambda}}^{o}\left\{\exp \left(-\frac{1}{\lambda^{2}}\left[\frac{1}{2}(A x, x)+F(A x)\right]\right) \exp \left(\frac{K_{1}}{\lambda^{2-\alpha}}(x, x)^{\alpha / 2}-\frac{1}{\lambda}(x, x)\right)\right\}
$$

which by Lemma $2.6(a)$ is

$$
\leqq K_{4} E_{x \in J_{2}^{\lambda}}^{o}\left\{\exp \left(K_{1}(x, x)^{\alpha / 2} / \lambda^{2-\alpha}-(x, x) / \lambda\right)\right\}
$$


The Cauchy-Schwarz inequality implies

$$
E_{2} \leqq K_{4}\left(E_{x}^{\rho}\left\{\exp \left(2 K_{1} / \lambda^{2-\alpha}-2(x, x) / \lambda\right)\right\}\right)^{1 / 2}\left(P\left(J_{2}^{\lambda}\right)\right)^{1 / 2} .
$$

By hypothesis we have $P\left(J_{2}^{\lambda}\right) \leqq \exp \left(-\delta^{2} / 4 \lambda^{2}\right)$. This inequality and Lemma 2.9 then give

$$
E_{2} \leqq 2 K_{4} \exp \left(\frac{K_{1}^{2 /(2-\alpha)}}{\lambda^{(2-\alpha) / 2}}\right) \exp \left(-\frac{\delta^{2}}{8 \lambda^{2}}\right)
$$

Again using (4.10) and (4.11) we obtain

$$
\begin{aligned}
E_{3} \leqq K_{4} E_{x \in J_{3}^{\lambda}}^{o}\left\{\operatorname { e x p } \left(-\frac{1}{\lambda^{2}}\left[\frac{1}{2}(A x, x)\right.\right.\right. & F(A x)]) \\
& \left.\times \exp \left(\frac{-K_{1}(x, x)^{\alpha / 2}}{\lambda^{2-\alpha}}\right) \exp \left(-\frac{(x, x)}{\lambda}\right)\right\}
\end{aligned}
$$

Setting $y=A x$ we see that $\frac{1}{2}(A x, x)+F(A x)=\frac{1}{2}\left(A^{-1} y, y\right)+F(y)$, while $x \in J_{3}^{\lambda}$ implies $R \geqq\left\|y-x^{*}\right\|>\delta / 2$. From Lemma 2.5 we then have

$$
\frac{1}{2}(A x, x)+F(A x)>\theta\left(\frac{1}{2} \delta\right)>0, \quad x \in J_{3}^{\lambda} .
$$

It follows from Lemma 2.9 that

$$
\begin{aligned}
E_{3} & \leqq K_{4} \exp \left(-\frac{1}{\lambda^{2}} \theta\left(\frac{1}{2} \delta\right)\right) E_{x}^{\rho}\left\{\exp \left(-\frac{K_{1}}{\lambda^{2-\alpha}}(x, x)^{\alpha / 2}-\frac{1}{\lambda}(x, x)\right)\right\} \\
& \leqq 2 K_{4} \exp \left(-\frac{\theta\left(\frac{1}{2} \delta\right)}{\lambda^{2}}\right) \exp \left(\frac{K_{1}^{2 /(2-\alpha)}}{\lambda^{(2-\alpha) / 2}}\right)
\end{aligned}
$$

If $x \in J_{4}^{\lambda}$, then $\|A x\| \leqq R+\left\|x^{*}\right\|$. Therefore, (4.9) shows that

$$
E_{4} \leqq K_{3} \exp c_{3}\left(R+\left\|x^{*}\right\|\right) E_{x \in J_{4}^{\lambda}}^{o}\left\{\exp \left(\lambda^{2}\|x\|^{2}+2 c_{3} \lambda\left(R+\left\|x^{*}\right\|\right)\|x\|\right)\right.
$$

$$
\left.\times \exp \left(-\frac{1}{\lambda^{2}}\left[\left(\frac{1}{2} A x+\lambda x, x\right)+F(A x+\lambda x)\right]\right)\right\}
$$

By the hypothesis on $F$, Lemma 2.2(b), (c) and (4.4) we have

$$
\frac{1}{2}(A x+\lambda x, x)+F(A x+\lambda x) \geqq \frac{1}{2}\left(1-c_{1} / \rho_{1}\right)[(A x, x)+\lambda(x, x)]-c_{2} \geqq-c_{2} .
$$

Therefore (4.15) shows

$E_{4} \leqq K_{3} \exp \left(c_{3}\left(R+\left\|x^{*}\right\|\right)^{2}\right) \exp \left(c_{2} / \lambda^{2}\right) E_{x \in J_{4}^{\lambda}}^{o}\left\{\exp \left(c_{3} \lambda^{2}\|x\|^{2}+2 c_{3} \lambda\left(R+\left\|x^{*}\right\|\right)\|x\|\right)\right\}$

which by the Cauchy-Schwarz inequality is

$$
\begin{aligned}
\leqq K_{3} \exp \left(c_{3}\left(R+\left\|x^{*}\right\|\right)^{2}\right) \exp \left(\frac{c_{2}}{\lambda^{2}}\right)\left[E _ { x } ^ { o } \left\{\exp \left(2 c_{3} \lambda^{2}\|x\|^{2}\right)\right.\right. \\
\left.\left.\times \exp \left(4 c_{3}\left(R+\left\|x^{*}\right\|\right)\|x\|\right)\right\}\right]^{1 / 2}\left[P\left(J_{4}^{\lambda}\right)\right]^{1 / 2}
\end{aligned}
$$


Using the hypothesis on the distribution of the supremum and (4.4) we obtain

$$
\begin{aligned}
E_{4} \leqq K_{3} \exp \left(c_{3}\left(R+\left\|x^{*}\right\|\right)^{2}\right) \exp & \left(-\frac{1}{\lambda^{2}}\left(\frac{R^{2}}{2}-c_{2}\right)\right) \\
\times & {\left[E_{x}^{o}\left\{\exp \left(\frac{\gamma}{2}\|x\|^{2}+2\left(\gamma c_{3}\right)^{1 / 2}\left(R+\left\|x^{*}\right\|\right)\|x\|\right)\right\}\right]^{1 / 2} }
\end{aligned}
$$

By the assumption on the supremum we see that the right hand side is finite. Letting

$$
K_{5}=K_{3} \exp \left(c_{3}\left(R+\left\|x^{*}\right\|\right)\right)\left[E_{x}^{o}\left\{\exp \left(\gamma / 2\left\|x^{*}\right\|^{2}\right) \exp \left(2\left(\gamma c_{3}\right)^{1 / 2}\left(R+\left\|x^{*}\right\|\right)\|x\|\right)\right\}\right]^{1 / 2}
$$

we have

$$
E_{4} \leqq K_{5} \exp \left(-\left[\gamma R^{2} / 2-c_{2}\right] / \lambda^{2}\right) \leqq K_{5} \exp \left(-1 / \lambda^{2}\right),
$$

by definition of $R$.

It follows from (4.9) and Lemma 2.2(d) that

$$
\begin{aligned}
E_{5} \leqq E_{x \in J_{5}}^{\rho}\left\{\exp \left(c_{3} \lambda^{2}\|x\|^{2}+2 \lambda c_{3}\left(\frac{M}{\rho_{1}}\right)^{1 / 2}\|x\|^{3 / 2}\right)\right. \\
\left.\quad \times \exp \left(-\frac{1}{\lambda^{2}}\left[\left(\frac{1}{2} A x+\lambda x, x\right)+F(A x+\lambda x)-\lambda^{2}\|A x\|^{2}\right]\right)\right\}
\end{aligned}
$$

which by (4.4) and (2.6) is

$$
\begin{aligned}
& \leqq \exp \left(\frac{c_{2}}{\lambda^{2}} K_{3}\right) E_{x \in J_{5}}^{o}\left\{\exp \left(\frac{\gamma}{2}\|x\|^{2}+\left(\frac{c_{3} \gamma M}{\rho_{1}}\right)^{1 / 2}\|x\|^{3 / 2}\right)\right. \\
& \left.\times \exp \left(-\frac{1}{\lambda^{2}} \frac{1}{2}\left(1-\frac{c_{1}}{\rho_{1}}\right)(A x, x)\right)\right\}
\end{aligned}
$$

If $x \in J_{5}$, then by Lemma 2.2(a) we have

$$
\begin{aligned}
R \leqq\left\|A x-x^{*}\right\| & =\left\|A^{1 / 2}\left(A^{1 / 2} x-A^{-1 / 2} x^{*}\right)\right\| \\
& \leqq M^{1 / 2}\left(A^{1 / 2} x-A^{-1 / 2} x^{*}, A^{1 / 2} x-A^{-1 / 2} x^{*}\right)^{1 / 2}
\end{aligned}
$$

which shows that

$$
(A x, x)^{1 / 2}=\left(A^{1 / 2} x, A^{1 / 2} x\right)^{1 / 2} \geqq R / M^{1 / 2}-\left(A^{-1 / 2} x^{*}, A^{-1 / 2} x^{*}\right)^{1 / 2} .
$$

Since $H\left(x^{*}\right)=0$ and $F(x) \geqq-\frac{1}{2} c_{1}(x, x)-c_{2}$ we see that

$$
0=H\left(x^{*}\right)=\frac{1}{2}\left(A^{-1 / 2} x^{*}, A^{-1 / 2} x^{*}\right)+F\left(x^{*}\right) \geqq \frac{1}{2}\left(\rho_{1}-c_{1}\right)\left(x^{*}, x^{*}\right)-c_{2},
$$

therefore,

$$
\left(A^{-1 / 2} x^{*}, A^{-1 / 2} x^{*}\right)=-2 F\left(x^{*}\right) \leqq c_{1}\left(x^{*}, x^{*}\right)+2 c_{2} \leqq 4 \rho_{1} c_{2} /\left(\rho_{1}-c_{1}\right) .
$$

Combining this last inequality with (4.17) we obtain

$$
(A x, x)^{1 / 2} \leqq R / M^{1 / 2}-\left(4 \rho_{1} c_{2} /\left(\rho_{1}-c_{1}\right)\right)^{1 / 2} .
$$


From (4.18) we then have

$$
\begin{aligned}
E_{5} \leqq \exp \left(-\frac{1}{\lambda^{2}}\left\{\frac{1}{2}\left(1-\frac{c_{1}}{\rho_{1}}\right)\left[\frac{R}{M^{1 / 2}}-\left(\frac{4 \rho_{1} c_{2}}{\rho_{1}-c_{1}}\right)^{1 / 2}\right]^{2}\right\}\right) \exp \left(\frac{c_{2}}{\lambda^{2}}\right) \\
\times K_{3} E_{x}^{o}\left\{\exp \left(\frac{\gamma}{2}\|x\|^{2}+\left(\frac{c_{3} \gamma M}{\rho_{1}}\right)^{1 / 2}\|x\|^{3 / 2}\right)\right\}
\end{aligned}
$$

Again by (2.8) the right hand side is finite.

Letting

$$
K_{6}=K_{3} E_{x}^{o}\left\{\exp \left((\gamma / 2)\|x\|^{2}+\left(c_{3} \gamma M / \rho_{1}\right)^{1 / 2}\|x\|^{3 / 2}\right)\right\}
$$

we obtain

$$
E_{5} \leqq K_{6} \exp \left(-\frac{1}{\lambda^{2}}\left\{\frac{1}{2}\left(1-\frac{c_{1}}{\rho_{1}}\right)\left(\frac{R}{M^{1 / 2}}-\left(\frac{4 \rho_{1} c_{2}}{\rho_{1}-c_{1}}\right)^{1 / 2}\right)^{2}-c_{2}\right\}\right)
$$

which by definition of $R$ gives

$$
E_{5} \leqq K_{6} \exp \left(-1 / \lambda^{2}\right) .
$$

Combining (4.7), (4.8), (4.12), (4.14), (4.16) and (4.19) we obtain

$$
\frac{E_{1}}{E} \leqq \frac{1}{5} \varepsilon
$$

$$
\begin{aligned}
& \frac{E_{2}}{E} \leqq \frac{2 K_{4}}{P\left(B_{\beta}\right)} \exp \left(-\frac{1}{\lambda^{2}}\left\{\frac{\gamma \delta^{2}}{8}-2 \eta-\lambda^{\alpha / 2} K_{1}^{2 /(2-\alpha)}\right\}\right) \\
& \frac{E_{3}}{E} \leqq \frac{2 K_{4}}{P\left(B_{\beta}\right)} \exp \left(-\frac{1}{\lambda^{2}}\left\{\theta\left(\frac{1}{2} \delta\right)-2 \eta-\lambda^{\alpha / 2} K_{1}^{2 /(2-\alpha)}\right\}\right) \\
& \frac{E_{4}}{E} \leqq \frac{K_{5}}{P\left(B_{\beta}\right)} \exp \left(-\frac{1}{\lambda^{2}}\left\{1-2 \eta-\lambda^{\alpha / 2} K_{1}^{2 /(2-\alpha)}\right\}\right) \\
& \frac{E_{5}}{E} \leqq \frac{K_{6}}{P\left(B_{\beta}\right)} \exp \left(-\frac{1}{\lambda^{2}}\left\{1-2 \eta-\lambda^{\alpha / 2} K_{1}^{2 /(2-\alpha)}\right\}\right) .
\end{aligned}
$$

From (4.5) we see that by choosing $\lambda$ sufficiently small, the coefficient of $-1 / \lambda^{2}$ in each of the exponentials (4.20)-(4.24) is strictly positive. This proves the theorem.

5. Applications to Hammerstein integral equations. Hammerstein equations are nonlinear integral equations of the form

$$
x(\sigma)+\int_{0}^{t} \rho(\sigma, \tau) f(\tau, x(\tau)) d \tau=0
$$

where $\rho(\sigma, \tau), 0 \leqq \sigma, \tau \leqq t$, is a square integrable (in the square $0 \leqq \sigma, \tau \leqq t$ ), symmetric, positive definite kernel and $f(\tau, u), 0 \leqq \tau \leqq t,-\infty<u<\infty$, is a real valued function. 
In this section we shall demonstrate a close connection between Theorem 4.1 and the theory of these equations. In fact, under certain conditions, Theorem 1 gives a closed form solution of (5.1). From two conditions, found by Hammerstein, which insure that (5.1) has a unique solution, we are able to give sufficient conditions insuring that the functional $H(x)=\frac{1}{2}\left(A^{-1 / 2} x, A^{-1 / 2} x\right)+F(x)$ has a unique global minimum.

When the kernel is that of Brownian motion (i.e. $\rho(\sigma, \tau)=\min (\sigma, \tau))$, it is possible to relate the solution of (5.1) to the Green's function of a linear parabolic equation through the Feynman-Kac formula [9]-[11].

Unless explicitly stated, we assume that $\rho(\sigma, \tau), 0 \leqq \sigma, \tau \leqq t$, is a continuous, positive definite, symmetric kernel (covariance function), and that $F(x)$ is a real valued functional defined on $C$ and continuous in the topology of $C$.

Whenever it exists, we shall call the derivative of $F$ with respect to $x$ at the point $\tau$, $0 \leqq \tau \leqq t, \delta F(x) / \delta x(\tau)$, the Fréchet-Volterra derivative of $F$ at $x$ (see $\S 2$ ).

By a standard variational argument we obtain

LEMMA 1. Let $H(x)=\frac{1}{2}\left(A^{-1 / 2} x, A^{-1 / 2} x\right)+F(x)$ have a minimum at the point $x^{*}$. If $F(x)$ has a Fréchet-Volterra derivative in a neighborhood $N$ of $x^{*}$ then $x^{*}$ satisfies the nonlinear integral equation

$$
x(\sigma)+\int_{0}^{t} \rho(\sigma, \tau) \frac{\delta F(x)}{\delta x(\tau)} d \tau=0, \quad x \in N
$$

Lemma 1 immediately implies

LEMMA 2. Let $\rho(\sigma, \tau)$ and $F(x)$ satisfy the conditions of Theorem 4.1. If, in addition, $F(x)$ has a Fréchet-Volterra derivative in a neighborhood $N$ of $x^{*}$, then

$$
\lim _{\lambda \rightarrow 0} \frac{E_{x x}^{\rho}\left\{\lambda x(\sigma) \exp \left(-F(\lambda x) / \lambda^{2}\right)\right\}}{E_{x}^{\rho}\left\{\exp \left(-F(\lambda x) / \lambda^{2}\right)\right\}}=x^{*}(\sigma)
$$

is a solution of the Hammerstein equation

$$
x(\sigma)+\int_{0}^{t} \rho(\sigma, \tau) \frac{\delta F(x)}{\delta x(\tau)} d \tau=0, \quad x \in N
$$

Another way of obtaining Lemma 2 without appealing to Lemma 1 is through the integration by parts formula for Gaussian processes which states that if $G(x)$ is integrable and if $G$ has a Fréchet-Volterra derivative $\delta G(x) / \delta x(\tau)$ satisfying certain conditions [13, p. 920], [14], then

$$
E_{x}^{\rho}\{x(\sigma) G(x)\}=E_{x}^{\rho}\left\{\int_{0}^{t} \rho(\sigma, \tau) \frac{\delta G(x)}{\delta x(\tau)} d \tau\right\}
$$


We then have, assuming that $F$ has a Fréchet-Volterra derivative for each sample path $x$ and that the conditions of Theorem 4.1 are satisfied,

$$
\begin{aligned}
& x^{*}(\sigma)=\lim _{\lambda \rightarrow 0} \frac{E_{x}^{o}\left\{\lambda x(\sigma) \exp \left(-F(\lambda x) / \lambda^{2}\right)\right\}}{E_{x}^{\rho}\left\{\exp \left(-F(\lambda x) / \lambda^{2}\right)\right\}} \\
& =\lim _{\lambda \rightarrow 0} \frac{E_{x}^{\rho}\left\{\int_{0}^{t} \rho(\sigma, \tau) \frac{\delta F(x)}{\delta x(\tau)} d \tau \exp \left(-F(\lambda x) / \lambda^{2}\right)\right\}}{E_{x}^{o}\left\{\exp \left(-F(\lambda x) / \lambda^{2}\right)\right\}} \\
& =\int_{0}^{t} \rho(\sigma, \tau) \frac{\delta F\left(x^{*}\right)}{\delta x(\tau)} d \tau
\end{aligned}
$$

the last step following from the application of Theorem 4.1 to the functional

$$
\int_{0}^{t} \rho(\sigma, \tau) \frac{\delta F(x)}{\delta x(\tau)} d \tau
$$

We shall now specialize to functionals of the form

$$
F(x)=\int_{0}^{t} V(\tau, x(\tau)) d \tau
$$

where $V(\tau, u), 0 \leqq \tau \leqq t,-\infty<u<\infty$, is a real valued function continuous in $u$, and integrable as a function of $\tau$ for each fixed $u$.

For functionals of this type we can give conditions directly on $V(\tau, u)$ that will ensure that the hypotheses of Theorem 4.1 are satisfied.

If $V(\tau, u) \geqq-\frac{1}{2} c_{1} u^{2}-c_{2}, c_{1}<\rho_{1}$, then it is easy to see that

$$
F(x) \geqq-\frac{1}{2} c_{1} \int_{0}^{t} x^{2}(\tau) d \tau-c_{2}, \quad c_{1}<\rho_{1} .
$$

If for $\left|u_{1}\right|,\left|u_{2}\right|<2 R$ we have

$$
\left|V\left(\tau, u_{2}\right)-V\left(\tau, u_{1}\right)\right| \leqq K\left|u_{2}-u_{1}\right|^{\alpha}, \quad 0<\alpha \leqq 1,
$$

then for $\|x\|,\|x+y\|<2 R$ it follows that

$$
\begin{aligned}
|F(x+y)-F(y)| & \leqq \int_{0}^{t}|V(\tau, x(\tau)+y(\tau))-V(\tau, x(\tau))| d \tau \\
& \leqq K \int_{0}^{t}|y(\tau)|^{\alpha} d \tau \leqq K\left(\int_{0}^{t}|y(\tau)|^{2} d \tau\right)^{\alpha / 2} t^{2 /(2-\alpha)} .
\end{aligned}
$$

Suppose $V(\tau, u)$ has a derivative, $V^{\prime}(\tau, u)$, with respect to $u,-\infty<u<\infty$, such that $V^{\prime}(\tau, x(\tau)) \in L^{2}$ for each $x \in C$. It then follows directly from the definition of the Fréchet-Volterra derivative that

$$
F(x)=\int_{0}^{t} V(\tau, x(\tau)) d \tau
$$

has a Fréchet-Volterra derivative, $\delta F(x) / \delta x(\tau)$, given by

$$
\delta F / \delta x(\tau)=V^{\prime}(\tau, x(\tau)), \quad x \in C .
$$


Lemma 1 then shows that the Euler-Lagrange equation of the functional

$$
H(x)=\frac{1}{2}\left(A^{-1 / 2} x, A^{-1 / 2} x\right)+\int_{0}^{t} V(\tau, x(\tau)) d \tau
$$

is the Hammerstein integral equation

$$
x(\sigma)+\int_{0}^{t} \rho(\sigma, \tau) V^{\prime}(\tau, x(\tau)) d \tau=0 .
$$

For the remainder of this section we shall assume that $V(\tau, u), 0 \leqq \tau \leqq t,-\infty$ $<u<\infty$ is, for each $u$, an integrable function of $\tau, 0 \leqq \tau \leqq t$, and that for $-\infty<u<\infty$, $V(\tau, u)$ has a derivative with respect to $u, V^{\prime}(\tau, u)$, such that $V^{\prime}(\tau, x(\tau)) \in L^{2}$ for each $x \in C$. These conditions will ensure $F(x)=\int_{0}^{t} V(\tau, x(\tau)) d \tau$ is well defined for each $x \in C$, and that at any point $x^{*}$ at which $H(x)$ assumes a minimum, $x^{*}$ will satisfy (5.4).

From two conditions on $V^{\prime}(\tau, u)$ which, as Hammerstein has shown, imply that (5.4) has a unique solution, we shall obtain conditions ensuring that $H(x)$ has a unique global minimum.

LEMMA 3. Assume $V(\tau, u), 0 \leqq \tau \leqq t,-\infty<u<\infty$, satisfies

$$
V(\tau, u) \geqq-\frac{1}{2} c_{1} u^{2}-c_{2}, \quad c_{1}<\rho_{1},-\infty<u<\infty, c_{2} \text { real. }
$$

Let $R$ have the meaning assigned in Theorem 4.1 and let $x^{*}$ denote a point at which $H(x)$ assumes its global minimum value $\left({ }^{4}\right)$.

Suppose $V^{\prime}(\tau, u)$ satisfies any one of the following conditions:

(5.6) $\left|V^{\prime}\left(\tau, u_{2}\right)-V^{\prime}\left(\tau, u_{1}\right)\right| \leqq K_{1}\left|u_{2}-u_{1}\right|, K_{1}<\rho_{1},\left|u_{1}\right|+\left\|x^{*}\right\| \leqq R,\left|u_{2}\right|+\left\|x^{*}\right\|$ $\leqq R$.

(5.6) a $\left|V^{\prime}\left(\tau, u_{2}\right)-V^{\prime}\left(\tau, u_{1}\right)\right| \leqq K_{1}\left|u_{2}-u_{1}\right|, K_{1}<\rho_{1},-\infty<u_{1}, u_{2}<\infty$.

(5.7) $V^{\prime}(\tau, u)$ is a monotone function of $u,|u|+\left\|x^{*}\right\| \leqq R$, for each fixed $\tau \in[0, t]$.

$(5.7)_{a} V^{\prime}(\tau, u)$ is a monotone function of $u,-\infty<u<\infty$, for each fixed $\tau \in[0, t]$.

It then follows that $x^{*}$ is the only point at which

$$
H(x)=\frac{1}{2}\left(A^{-1 / 2} x, A^{-1 / 2} x\right)+\int_{0}^{t} V(\tau, x(\tau)) d \tau
$$

assumes its global minimum value in the sphere $\left\{x \in C:\left\|x-x^{*}\right\| \leqq R\right\}$.

Under $(5.6)_{\mathrm{a}}$ or $(5.7)_{\mathrm{a}}, x^{*}$ is the only point at which $H(x)$ assumes a relative minimum value $\left({ }^{5}\right)$.

Proof. It is shown in [2, pp. 211-212] that under (5.6) $)_{a}$ or $(5.7)_{a}$ that (5.4) has a unique solution. Moreover, from the proofs given in this reference it is not difficult to see that under (5.6) or (5.7) that (5.4) has only one solution in the sphere

(4) Such a point exists by Lemma 2.4 when $V(\tau, u)$ satisfies (5.5).

(5) Therefore, $H\left(x^{*}\right)$ is a global minimum value of $H(x)$. 
$\left\{x \in C:\left\|x-x^{*}\right\| \leqq R\right\}$. The discussion leading to (5.4) then shows that $H(x)$ has a unique minimum in the sphere $\left\{x \in C:\left\|x-x^{*}\right\| \leqq R\right\}$.

It may be of some interest to show that (5.7) $)_{\mathrm{a}}$ implies $x^{*}$ is unique, directly from the properties of $H(x)$. Without loss of generality we can assume that at any point $x^{*}$ at which $H(x)$ attains its global minimum, $H\left(x^{*}\right)=0$. Also note that from (5.4) it follows that $x^{*}$ satisfies

$$
\left(A^{-1} x^{*}\right)(\tau)=V^{\prime}\left(\tau, x^{*}(\tau)\right)
$$

Thus, using (5.8) and the mean value theorem we obtain for all $y \in D\left(A^{-1}\right)$

$$
\begin{aligned}
H\left(x^{*}+y\right) & =\frac{1}{2}\left(A^{-1}\left[x^{*}+y\right], x^{*}+y\right)+F\left(x^{*}+y\right) \\
& =\frac{1}{2}\left(A^{-1} y, y\right)+\int_{0}^{t}\left[V^{\prime}\left(\tau, x^{*}(\tau)+\xi y(\tau)\right)-V^{\prime}\left(\tau, x^{*}(\tau)\right)\right] y(\tau) d \tau, \\
& 0<\xi \leqq 1 .
\end{aligned}
$$

When $V^{\prime}(\tau, u)$ satisfies $(5.7)_{\mathrm{a}}$ then

$$
V^{\prime}\left(\tau, x^{*}(\tau)+\xi y(\tau)\right)-V^{\prime}\left(\tau, x^{*}(\tau)\right)
$$

and $y(\tau)$ have the same sign. Therefore the integral in (5.9) is strictly positive for $y \neq 0$ and the conclusion follows.

It is not clear to the author how to construct a similar proof using condition (5.6) a . However, when $K_{1}<\rho_{1} / 2$ this will follow from Lemma 2.2(b) since (5.6)a implies

$$
\int_{0}^{t}\left[V^{\prime}\left(\tau, x^{*}(\tau)+\xi y(\tau)\right)-V^{\prime}\left(\tau, x^{*}(\tau)\right)\right] y(\tau) d \tau \geqq-K_{1} \int_{0}^{t} y^{2}(\tau) d \tau .
$$

The next theorem is an immediate consequence of Lemma 2, (5.2) and (5.3).

THEOREM 1. If $V(\tau, u)$ satisfies (5.5) and $V^{\prime}(\tau, u)$ satisfies any one of the conditions (5.6), (5.6) $)_{a},(5.7),(5.7)_{a}$, then

$$
\lim _{\lambda \rightarrow 0} \frac{E_{x}^{o}\left\{\lambda x(\sigma) \exp \left(-1 / \lambda^{2} \int_{0}^{t} V(\tau, \lambda x(t)) d \tau\right)\right\}}{E_{x}^{o}\left\{\exp \left(-1 / \lambda^{2} \int_{0}^{t} V(\tau, \lambda x(\tau)) d \tau\right)\right\}}
$$

is a solution of the Hammerstein integral equation

$$
x(\sigma)+\int_{0}^{t} \rho(\sigma, \tau) V^{\prime}(\tau, x(\tau)) d \tau=0 .
$$

Under $(5.6)_{\mathrm{a}}$ or $(5.7)_{\mathrm{a}}$ it is the unique solution.

In proving the existence of solutions to (5.4), Hammerstein considered the functionals

$$
\sum_{i=1}^{n} \rho_{i} \eta_{i}^{2}+\int_{0}^{t} V\left(\tau, \sum_{i=1}^{n} \eta_{i} u_{i}(\tau)\right) d \tau
$$


with the assumption $V(\tau, u) \geqq-\frac{1}{2} c_{1} u^{2}-c_{2}$. This condition ensured that for each $n$ there exists a point $x_{n}^{*}(\cdot)=\sum_{i=1}^{n} \eta_{i, n}^{*} u_{i}(\cdot)$ at which (5.10) assumed its global minimum.

By differentiating (5.10) we see that $x_{n}^{*}$ satisfies

where

$$
x_{n}^{*}(\sigma)+\int_{0}^{t} \rho_{n}(\sigma, \tau) V^{\prime}\left(\tau, x_{n}^{*}(\tau)\right) d \tau=0
$$

$$
\rho_{n}(\sigma, \tau)=\sum_{i=1}^{n} \frac{u_{i}(\sigma) u_{i}(\tau)}{\rho_{i}}
$$

Hammerstein then showed that the sequence $\left\{x_{n}^{*}\right\}$ contained a subsequence $\left\{x_{n,}^{*}\right\}$ that converged uniformly to a solution of (5.4).

From the point of view taken in this paper, the assumption that $V(\tau, u) \geqq$ $-\frac{1}{2} c_{1} u^{2}-c_{2}$ with $c_{1}<\rho_{1}$, is an integrability condition which allows a solution of (5.4) to be represented in terms of Gaussian integrals. For functionals of the type $F(x)=\int_{0}^{t} V(\tau, x(\tau)) d \tau$ this is the appropriate integrability condition since if $c_{1} \geqq \rho_{1}$ then $\exp \left(-\int_{0}^{t} V(\tau, x(\tau)) d \tau\right)$ would not be integrable.

The sequence $\left\{x_{n}^{*}\right\}$ is clearly a Rayleigh-Ritz minimizing sequence of $H(x)$. Lemma 2.4 shows that not only this sequence but every minimizing sequence of $H(x)$ contains a subsequence that converges uniformly to a solution of (5.4). If there is a unique solution of (5.4) then every minimizing sequence of $H$ converges uniformly to that solution.

We now specialize the covariance function $\rho(\sigma, \tau)$ to the case of Brownian motion, where $\rho(\sigma, \tau)=\min (\sigma, \tau)$, and a closely related covariance function $T(\sigma, \tau)$ where

$$
\begin{aligned}
T(\sigma, \tau) & =\sigma(1-\tau / t), & & 0 \leqq \sigma \leqq \tau \leqq t, \\
& =\tau(1-\sigma / t), & & 0 \leqq \tau \leqq \sigma \leqq t .
\end{aligned}
$$

It is well known [3] that $T(\sigma, \tau)$ is the Green's function of the operator on $L^{2}$

$$
A^{-1}=-d^{2} / d \sigma^{2}
$$

where $D\left(A^{-1}\right)=\left\{x \in L^{2}: x\right.$ twice differentiable, $\left.\left(x^{\prime \prime}, x^{\prime \prime}\right)<\infty, x(0)=x(t)=0\right\}$.

The connection between Brownian motion and the Gaussian process generated by $T(\sigma, \tau)$ is contained in the formula

$$
E_{x}^{\min }\{G(x) \mid x(t)=\eta\}=E_{x}^{T}\{G(x(\cdot)+(\cdot) \eta / t)\}
$$

where if one side exists so does the other and both are equal [12].

Under certain conditions [9]-[11] on the functions $V(\tau, u)$ and $q(\tau)$ it can be shown that

$$
\begin{aligned}
u(\eta, t, \lambda, q)=\frac{\exp \left(-\eta^{2} / 2 t\right)}{\sqrt{ }(2 t)} E_{x}^{\min }\{\exp ( & \left.-\frac{1}{\lambda^{2}} \int_{0}^{t} V(\tau, x(\tau)) d \tau\right) \\
& \left.\times \exp \left(\frac{1}{\lambda} \int_{0}^{t} q(\tau) x(\tau) d \tau\right) \mid x(t)=\eta\right\}
\end{aligned}
$$


is the unique solution of

$$
\begin{aligned}
\frac{\partial u}{\partial t}=\frac{\lambda^{2}}{2} \frac{\partial^{2} u}{\partial \eta^{2}}+\frac{1}{\lambda^{2}}[V(\tau, \eta)-q(\tau) \eta] u & =0, \\
\lim _{\eta \rightarrow \infty} u(\eta, t, \lambda, q) & =0, \\
\lim _{t \rightarrow 0^{+}} u(\eta, t, \lambda, q) & =\delta(\eta) .
\end{aligned}
$$

Let us note that

$$
\begin{aligned}
& \lambda^{2} \frac{\delta u /(\delta q(\sigma))}{u} \\
& =\frac{E_{x}^{\min }\left\{\lambda x(\sigma) \exp \left(-1 / \lambda^{2} \int_{0}^{t} V(\tau, \lambda x(\tau)) d \tau+1 / \lambda \int_{0}^{t} q(\tau) x(\tau) d \tau\right) \mid x(t)=\eta\right\}}{E_{x}^{\min }\left\{\exp \left(-1 / \lambda^{2} \int_{0}^{t} V(\tau, \lambda x(\tau))+1 / \lambda \int_{0}^{t} q(\tau) x(\tau) d \tau\right) \mid x(t)=\eta\right\}} \\
& =\frac{E_{x}^{T}\left\{(\lambda x(\sigma)+\sigma \eta / t) \exp \left(-1 / \lambda^{2} \int_{0}^{t} V(\tau, \lambda x(\tau)+\tau \eta / t) d \tau+1 / \lambda \int_{0}^{t} q(\tau) x(\tau) d \tau\right)\right\}}{E_{x}^{T}\left\{\exp \left(-1 / \lambda^{2} \int_{0}^{t} V(\tau, \lambda x(\tau)+\tau \eta / t) d \tau+1 / \lambda \int_{0}^{t} q(\tau) x(\tau) d \tau\right)\right\}} \\
& 0 \leqq \sigma \leqq t
\end{aligned}
$$

Thus, if $q(\tau)$ and $V(\tau, u)$ are such that the conditions of Theorem 4.1 are fulfilled, then (5.14) shows that

$$
\lim _{\lambda \rightarrow 0} \lambda^{2} \frac{\delta u /(\delta q(\sigma))}{u}=x^{*}(\sigma)+\sigma \eta / t, \quad 0 \leqq \sigma \leqq \tau,
$$

where $x^{*}$ satisfies the Hammerstein integral equation

$$
x(\sigma)+\int_{0}^{t} T(\sigma, \tau)\left[V^{\prime}\left(\tau, x(\tau)+\frac{\tau}{t} \eta\right)+q(\tau)\right] d \tau=0,
$$

or $x^{*}(\sigma)+\sigma \eta / t$ satisfies the equation

$$
x(\sigma)+\int_{0}^{t} T(\sigma, \tau)\left[V^{\prime}(\tau, x(\tau))+q(\tau)\right] d \tau+\frac{\sigma}{t} \eta=0 .
$$

From (5.11) we see that $(5.15)$ is equivalent to the differential equation

$$
\begin{gathered}
-x^{\prime \prime}(\sigma)+V^{\prime}(\sigma, x(\sigma))+q(\sigma)=0, \quad 0 \leqq \sigma \leqq t, \\
x(0)=0, \quad x(t)=\eta .
\end{gathered}
$$

We have thus shown that

THEOREM 2. If $V(\tau, u)$ and $q(\tau)$ satisfy conditions ensuring the validity of the Feynman-Kac formula (5.13) ([9]-[11]) and are such that the functional

$$
F(x)=\int_{0}^{t}[V(\tau, x(\tau))-q(\tau) x(\tau)] d \tau
$$


satisfies the conditions of Theorem 4.1 (see Lemma 3), then

$$
\lim _{\lambda \rightarrow 0} \lambda^{2} \frac{\delta u /(\delta q(\sigma))}{u}, \quad 0 \leqq \sigma \leqq t,
$$

is the solution of

$$
\begin{gathered}
-x^{\prime \prime}(\sigma)+V^{\prime}(\sigma, x(\sigma))+q(\sigma)=0, \quad 0 \leqq \sigma \leqq t, \\
x(0)=0, \quad x(t)=\eta,
\end{gathered}
$$

where $u=u(\eta, t, \lambda, q)$ is the solution of

$$
\begin{gathered}
\frac{\partial u}{\partial t}=\frac{\lambda^{2}}{2} \frac{\partial^{2} u}{\partial \eta^{2}}+\frac{1}{\lambda^{2}}(V(\tau, \eta)-q(\tau) \eta) u=0, \\
\lim _{\eta \rightarrow \infty} u(\eta, t, \lambda, q)=0, \quad \lim _{t \rightarrow 0^{+}} u(\eta, t, \lambda, q)=\delta(\eta) .
\end{gathered}
$$

\section{BIBLIOGRAPHY}

1. M. Schilder, A Laplace asymptotic formula for Wiener integrals, Thesis, New York University, 1965.

2. F. G. Tricomi, Integral equations, Interscience, New York, 1957.

3. S. G. Mikhlin, The problem of the minimum of a quadratic functional, transl. by A. Feinstein, Holden-Day, San Francisco, Calif., 1965.

4. N. Dunford and J. T. Schwartz, Linear operators, Part 1, Interscience, New York, 1958.

5. D. E. Varberg, Some Radon-Nikodym derivatives associated with stochastic processes, Thesis, University of Minnesota, 1959.

6. E. E. Slutsky, Qualche proposizione relativa alla teoria delle funzioni aleatorie, Giorn. Ist. Ital. Attuari 8 (1937), 183-199.

7. Z. Ciesielski, Hölder conditions for realizations of Gaussian processes, Trans. Amer. Math. Soc. 99 (1961), 403-413.

8. Yu. V. Prohorov, Convergence of random processes and limit theorems in probability theory, Theor. Probability Appl. 1 (1956), 157-214.

9. R. H. Cameron, The generalized heat flow equation and a corresponding Poisson formula, Ann. of Math. (2) 59 (1954), 434-462.

10. A. J. Tingley, On a generalization of the Poisson formula for the solution of the heat flow equation, Proc. Amer. Math. Soc. 7 (1956), 846-851.

11. M. Kac, Probability and related topics in the natural sciences, Interscience, New York, 1957.

12. M. Pincus, Gaussian processes and Hammerstein integral equations, Thesis, New York University, 1965.

13. R. H. Cameron, The first variation of an indefinite Wiener integral, Proc. Amer. Math. Soc. 2 (1951), 914-924.

14. M.Pincus, The translation and related theorems for Gaussian processes, Proc. Amer. Math. Soc. (to appear).

Polytechnic INSTITUTE OF BROOKLyN, BROOKLYN, NEW YORK 